\title{
Diagnóstico clínico e radiográfico de luxação traumática da articulação atlanto-occipital em dois cães
}

\author{
[Clinical and radiographic diagnosis of traumatic dislocation of \\ the atlanto-occipital joint in two dogs] \\ B.M. Araújo ${ }^{1}$, M.L. Figueiredo ${ }^{2}$, A.C. Silva ${ }^{2}$, T.H.T. Fernandes ${ }^{2}$, E.A. Tudury ${ }^{3}$ \\ ${ }^{1}$ Aluno de pós-graduação - Universidade Federal Rural de Pernambuco - Recife, PE \\ ${ }^{2}$ Universidade Federal Rural de Pernambuco - Recife, PE
}

\begin{abstract}
RESUMO
A luxação da articulação atlanto-occipital é considerada uma afecção incomum no homem e nos animais. Radiografias laterais são recomendadas para o diagnóstico. No entanto, estão sujeitas a erros relacionados ao ângulo de radiação, ao alvo da imagem, à distância e à sobreposição óssea. Objetivou-se neste relato descrever os achados clínicos e radiográficos de dois cães com luxação traumática da articulação atlantooccipital que apresentavam tetraparesia, dor cervical cranial, incapacidade de elevar a cabeça e déficits de nervos cranianos. No primeiro animal, observaram-se deslocamento craniodorsal do processo articular do atlas em relação a um dos côndilos do occipital, ausência de sobreposição dos forames vertebrais laterais e sobreposição do côndilo do occipital ao processo articular do atlas, no lado direito, caracterizando uma luxação unilateral. No segundo animal, observou-se deslocamento craniodorsal dos processos articulares do atlas em relação aos côndilos do occipital, com sobreposição dos forames vertebrais laterais e ausência de visibilização dos côndilos do occipital em virtude da projeção cranial dos processos articulares do atlas em direção ao crânio, caracterizando luxação bilateral. Conclui-se que o exame radiográfico simples, nas projeções laterolateral e ventrodorsal, apesar da dificuldade de ser interpretado, é eficiente para confirmar o diagnóstico da luxação atlanto-occipital traumática, tanto a simétrica quanto a assimétrica.
\end{abstract}

Palavras-chave: junção craniocervical, luxação, critério radiodiagnóstico

\begin{abstract}
Atlanto-occipital luxation is considered rare in both humans and animals. Lateral radiographs are recommended for diagnosis, however, errors may occur related to the angle of radiation, image target, distance and overlapping of bone. Our objective is to report the clinical and radiographic findings in two dogs with traumatic atlanto-occipital luxation, which had tetraparesis, cranial neck pain, and inability to raise the head and cranial nerve deficits. The first animal had a cranio-dorsal dislocation of the articular process of the atlas in relation to one of the occipital condoles, with no overlapping of the transverse foramens or overlapping of the occipital condile in relation to the articular process of the atlas, on the right side, which characterizes a unilateral luxation. The second animal presented with a cranio-caudal dislocation of the articular processes of the atlas regarding the occipital condoles, with overlapping of the transverse foramens and inability to visualize the occipital condoles due to the cranial advancement of the articular processes of the atlas towards the skull, which characterizes a bilateral luxation. We conclude that a simple radiographic exam, in lateral and ventrodorsal projections, though difficult to interpret, is efficient in confirming a diagnosis of traumatic atlanto-occipital luxation, both symmetric and asymmetric.
\end{abstract}

Keywords: craniocervical junction, luxation, radiodiagnostic criteria

Recebido em 2 de março de 2012

Aceito em 4 de setembro de 2012

E-mail: bmaraujo85@hotmail.com 


\section{INTRODUÇÃO}

A luxação da articulação atlanto-occipital (A-O) foi descrita pela primeira vez em humanos em 1908 (Przybylski et al., 1996) e é considerada uma afecção incomum no homem e nos animais (Greenwood e Oliver, 1978; DeCamp et al., 1991; Przybylski et al., 1996; Lorenz e Kornegay, 2006; Buks et al., 2011). Na maioria dos casos está associada à morte, sendo raros os relatos de animais que sobrevivem ao trauma (Greenwood e Oliver, 1978; DeCamp et al., 1991; Rylander e Robles, 2007).

Nos poucos casos descritos na literatura, os animais foram atendidos devido a acidente com veículos automotivos, e os sinais clínicos variaram de dor no pescoço a coma transitório, progredindo para tetraparesia, cujas sequelas variaram de acordo com a gravidade da lesão (Greenwood e Oliver, 1978; DeCamp et al., 1991).

Radiografias laterais são recomendadas para o diagnóstico (Steffen et al., 2003). No entanto, estão sujeitas a erros relacionados ao ângulo da radiação, ao alvo da imagem, à distância e à sobreposição óssea (Pang et al., 2007a). Se existir a suspeita de luxação e o exame radiográfico comum não for conclusivo, recomenda-se a realização de tomografia computadorizada (TC) e ressonância magnética (RM) (Przybylski, et al., 1996; Steffen et al., 2003). A TC é um excelente meio de diagnóstico da luxação A-O, pois demonstra a orientação espacial da luxação e ainda a existência de fraturas não visíveis em radiografias convencionais (Steffen et al., 2003; Rylander e Robles, 2007). A RM é útil nesses casos, auxiliando na identificação de edema medular e/ou cerebral, possíveis hemorragias subaracnoideas (Przybylski et al., 1996) ou lesões de tecidos moles, principalmente a ruptura da membrana A-O (Pang et al., 2007b).

Os achados radiográficos de animais com luxação A-O incluem aumento da radiopacidade dos tecidos moles periarticulares, desalinhamento anatômico da articulação, com posição oblíqua das asas do atlas na projeção lateral, sobreposição dos processos articulares do atlas em relação aos côndilos do occipital na projeção lateral e possíveis fragmentos causados por avulsões ósseas (Greenwood e Oliver, 1978; Lappin e Dow, 1983; Steffen et al., 2003).

O prognóstico dos animais sobreviventes ao trauma varia de reservado a favorável, com recuperação satisfatória na maioria dos casos (Greenwood e Oliver, 1978; DeCamp et al., 1991; Steffen et al., 2003; Lorenz e Kornegay, 2006), embora possam ocorrer complicações pós-operatórias graves nas cirurgias realizadas nessa região (DeCamp et al., 1991; Dewey, 2006).

O objetivo deste trabalho foi descrever os sinais clínicos e achados radiográficos de dois cães com luxação atlanto-occipital, em virtude da incomum ocorrência na medicina veterinária.

\section{CASUÍSTICA}

Foram atendidos no Hospital Veterinário da Universidade Federal Rural de Pernambuco dois cães, sem raça definida, machos, sendo o primeiro de oito anos de idade e pesando $11 \mathrm{~kg}$ e o segundo de três anos de idade e pesando $8 \mathrm{~kg}$, que apresentavam síndrome neurológica multifocal (coluna cervical e tronco encefálico) após acidente automotivo há 10 e três dias, respectivamente. De acordo com os tutores, ambos os animais apresentavam fortes dores no pescoço e não andaram mais com os quatro membros desde o acidente.

No exame clínico, o primeiro animal encontravase em decúbito lateral e observaram-se mucosas hipocoradas, dispneia, sialorreia intensa, dor cervical grave, com assimetria das asas do atlas na palpação vertebral. No exame neurológico, foram constatados tetraparesia, ausência de propriocepção e reações posturais nos quatro membros, espasticidade dos membros torácicos, estrabismo ventrolateral no olho direito, diminuição da sensibilidade nasal e dos reflexos de deglutição e de retração do globo ocular bilateralmente, hipotônia da língua, atrofia da musculatura cervical (provavelmente de origem neurogênica), reflexos espinhais normais nos 
membros torácicos e aumentados nos membros pélvicos, presença do reflexo extensor cruzado nos membros pélvicos e torácicos, reflexo cutâneo do tronco presente em toda sua extensão, hiperpatia espinhal cervical cranial e presença de nocicepção (dor superficial) nos quatro membros.

O segundo animal apresentava-se em decúbito lateral, com tetraparesia grave, ausência de reações posturais e propriocepção nos quatro membros, presença de espasticidade em todos os membros, ausência de reação à ameaça bilateralmente e ao teste de sensibilidade nasal do lado esquerdo, reflexos espinhais aumentados com presença do reflexo extensor cruzado nos quatro membros e do reflexo de Babinski nos membros pélvicos, reflexo cutâneo do tronco presente em toda sua extensão e nocicepção preservada nos quatro membros.

Diante dos dados da anamnese e dos achados do exame clínico geral e neurológico, suspeitou-se em ambos os casos de uma lesão neurológica multifocal traumática, atingindo medula espinhal cervical e tronco encefálico (região pontinobulbar), solicitando-se radiografias da articulação atlanto-occipital e coluna cervical, nas projeções lateral e ventrodorsal.

Devido à instabilidade vertebral, os animais foram colocados em uma superfície rígida, sendo presos com fitas adesivas para evitar a movimentação da cabeça e coluna cervical e submetidos ao procedimento cirúrgico de redução aberta e estabilização da articulação A-O.

No primeiro animal, observou-se, no exame radiográfico (na projeção lateral), deslocamento craniodorsal do processo articular do atlas em relação a um dos côndilos do occipital, com ausência de sobreposição dos forames vertebrais laterais. $\mathrm{Na}$ projeção ventrodorsal, observou-se sobreposição do côndilo do occipital em relação ao processo articular do atlas, no lado direito, caracterizando uma luxação unilateral da articulação A-O (Fig. 1).
No segundo animal, observou-se na projeção lateral deslocamento craniodorsal dos processos articulares do atlas em relação aos côndilos do occipital, com sobreposição dos forames vertebrais laterais. Na projeção ventrodorsal, observou-se ausência de visibilização dos côndilos do occipital em virtude da projeção cranial dos processos articulares do atlas em direção ao crânio, caracterizando luxação bilateral da articulação A-O (Fig. 2).

Os dois animais foram a óbito dois dias e seis horas após o procedimento cirúrgico de redução aberta e estabilização, respectivamente.

\section{DISCUSSÃO}

O diagnóstico desta afecção da articulação A-O foi obtido por meio da correlação dos dados da anamnese, dos achados do exame clínico geral e neurológico e do exame radiográfico simples, que foram suficientes para confirmar o diagnóstico (Steffen et al., 2003).

O acidente que resultou na luxação foi causado por atropelamento, sendo esta a única etiologia descrita até o momento nos relatos em cães, que foi o acidente com veículos automotivos (Greenwood e Oliver, 1978; DeCamp et al., 1991).

Os achados do exame neurológico de tetraparesia, com ausência de propriocepção nos quatro membros, com todos os reflexos espinhais preservados, presença do reflexo extensor cruzado nos quatro membros, alteração das respostas de alguns reflexos dos nervos cranianos (devido à proximidade do tronco encefálico à articulação atlanto-occipital) e hiperpatia cervical cranial ajudaram na localização neuroanatômica da lesão, confirmada por meio do exame radiográfico. No entanto, pela falta de recursos, não foi possível avaliar a presença de microfraturas, edemas, hemorragias subaracnoideas ou lesões de tecidos moles, que podem ser visibilizados por meio de exames de TC e RM (Przybylski et al., 1996; Steffen et al., 2003; Pang et al., 2007b; Rylander e Robles, 2007). 

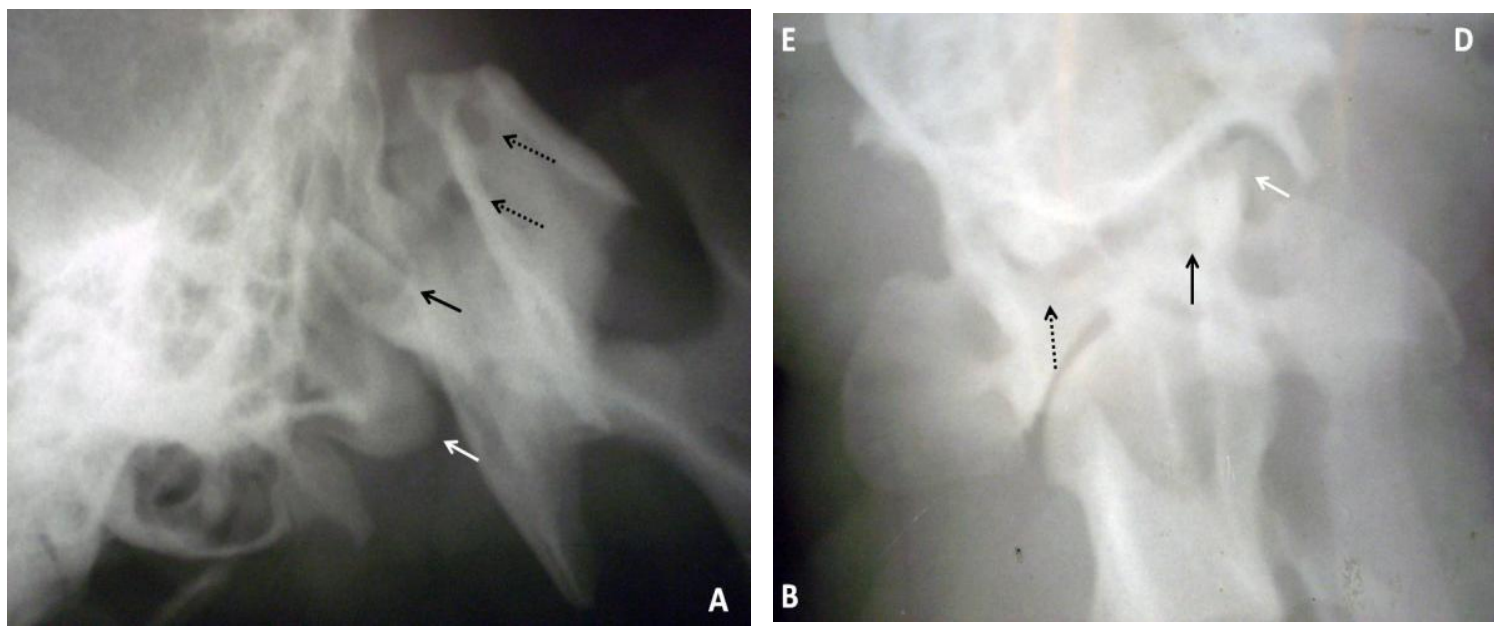

Figura 1. Imagens radiográficas da articulação atlanto-occipital, na projeção lateral (A), observando-se deslocamento craniodorsal do processo articular do atlas (seta preta) em relação ao côndilo occipital (seta branca), com ausência de sobreposição dos forames vertebrais laterais (setas pontilhadas). Projeção ventrodorsal (B), observando-se sobreposição do côndilo do occipital em relação ao processo articular do atlas (seta preta), com deslocamento cranial do processo articular do atlas em direção ao crânio, no lado direito (D) (seta branca). Notar a articulação normal do processo articular do atlas com o côndilo occipital esquerdo (seta pontilhada).
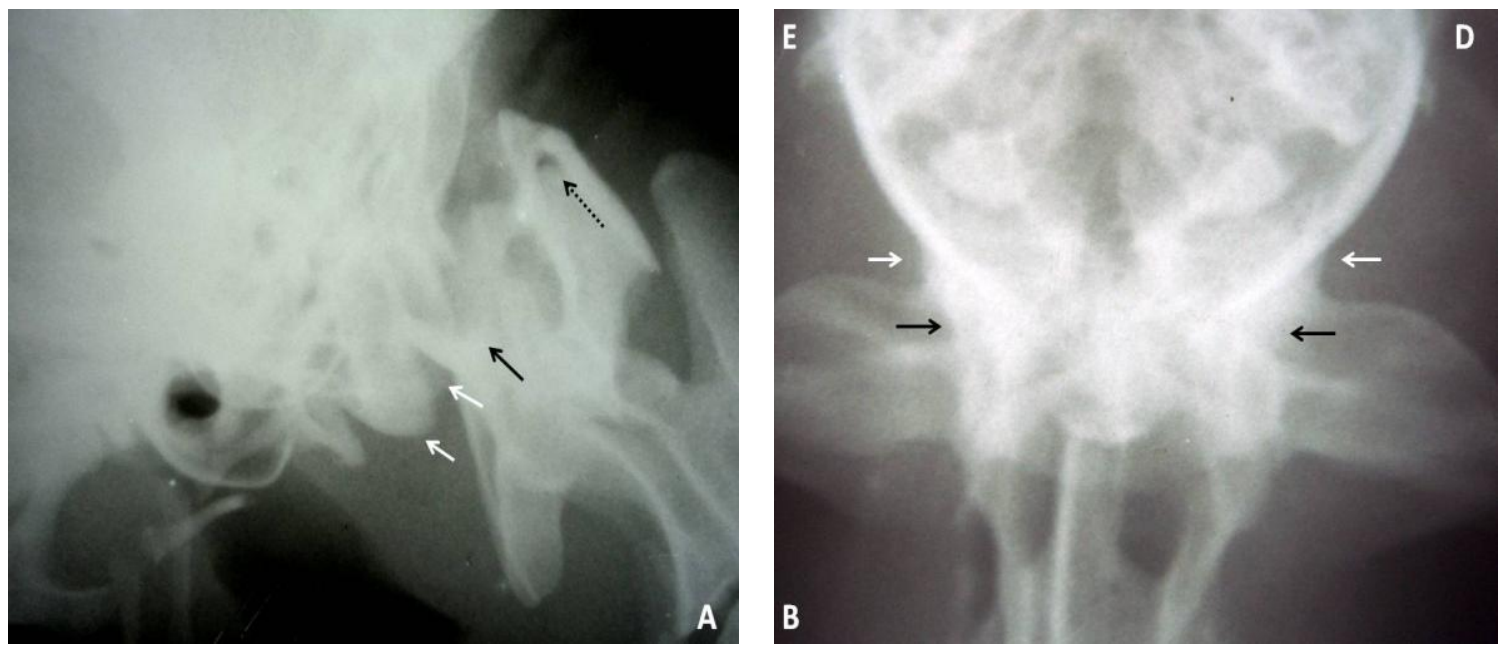

Figura 2. Imagens radiográficas da articulação atlanto-occipital, na projeção lateral (A), observando-se deslocamento craniodorsal dos processos articulares do atlas (seta preta) em relação aos côndilos do occipital (setas brancas), com sobreposição dos forames vertebrais laterais (seta pontilhada). Projeção ventrodorsal (B), observando-se ausência de visibilização dos côndilos do occipital (setas pretas) em virtude da projeção cranial dos processos articulares do atlas em direção ao crânio (setas brancas).

No primeiro animal, a lateralidade da lesão evidenciou-se clinicamente por estrabismo ventrolateral do olho direito e pode ser visibilizada por meio da ausência de sobreposição dos forames vertebrais laterais na projeção lateral, pelo deslocamento assimétrico do processo articular do atlas em direção ao crânio do lado direito e pela visibilização da articulação normal do côndilo do occipital com o processo articular do atlas no lado esquerdo na projeção ventrodorsal.

A simetria da lesão do segundo animal foi detectada pela sobreposição dos forames 
vertebrais laterais na projeção lateral e pelo deslocamento em conjunto dos processos articulares do atlas em direção ao crânio, não sendo possível, desta forma, a visibilização de ambos os côndilos do occipital que se encontravam sobrepostos pelos processos articulares do atlas na projeção ventrodorsal.

Em humanos, por meio da utilização de TC, Pang et al. (2007 a,b) descreveram um método de detecção de deslocamento A-O mediante a medição do intervalo de côndilo-C1 (CCI) e definiram o intervalo normal de $2 \mathrm{~mm}$. Consequentemente, a separação de $4 \mathrm{~mm}$ ou qualquer distância acima desta é considerada anormal. Os autores compararam esses valores com outros testes-padrão, bem como com os indicadores não padrão, e descobriram que a medição da distância do CCI por TC é extremamente sensível para o diagnóstico de deslocamento atlanto-occipital, ocasionando maiores taxas de sobrevivência em relação a estudos que utilizam outros meios de diagnósticos.

Seguindo os estudos de Pang et al. (2007a,b) em humanos, métodos devem ser padronizados na medicina veterinária no intuito de aumentar a sensibilidade dos diagnósticos, uma vez que as possíveis ocorrências dessa afecção podem ser mascaradas por métodos não adequados de identificação e falta de experiência de alguns profissionais, ocasionando a atual escassez de relatos de casos em animais, provavelmente por serem subdiagnosticados. De acordo com Przybylski et al. (1996), o diagnóstico de luxação traumática da articulação A-O é muitas vezes não atingido nos setores de emergência, e os métodos atuais para avaliar a integridade da articulação A-O na radiografia cervical não conseguem identificar todos os pacientes com essa lesão.

Os animais foram a óbito provavelmente por complicações respiratórias. De acordo com Steffen et al. (2003), em cirurgias na medula espinhal superior, o edema e a hemorragia podem se estender em direção ao tronco encefálico, produzindo déficits das funções bulbares (respiratórias e cardiovasculares) e dos nervos cranianos. Dewey (2006) ainda menciona que, no acesso cirúrgico ventral à articulação atlantoaxial, ocasionalmente ocorrem complicações pós-operatórias envolvendo o sistema respiratório.

\section{CONCLUSÃO}

Conclui-se que a luxação traumática da articulação atlanto-occipital é uma afecção grave em cães, podendo resultar em déficits neurológicos severos e complicações póscirúrgicas, sendo passível de diagnóstico, quando uni ou bilateral, por meio de exames radiográficos simples nas projeções laterolateral e ventrodorsal.

\section{REFERÊNCIAS}

BUKS, I.; SNELLING, S.R.; YATES, G.D. Ventral fixation of chronic atlanto-occipital luxation in a dog. J. Small. Anim. Pract., v.52, p.497-500, 2011.

DECAMP, C.E.; SCHIRMER, R.G.; STICKLER, R.L. Traumatic atlantooccipital subluxation in a dog. J. Am. Anim. Hosp. Assoc., v.27, p.415-418, 1991.

DEWEY, W. Mielopatias: doenças da medula espinhal. In: Neurologia de cães e gatos. Guia prático. São Paulo: Roca, 2006. p.163-196.

GREENWOOD, K.M.; OLIVER, J.E. Traumatic atlanto-occipital dislocation in two dogs. J. Am. Vet. Med. Assoc., v.173, p.1324-1327, 1978.

LAPPIN, M.R.; DOW, S.W. Traumatic atlantooccipital luxation in a cat. Vet. Surg., v.12, p.3032, 1983.

LORENZ, M.D.; KORNEGAY, J.N. Tetraparesia, hemiparesia e ataxia. In: Neurologia veterinária. São Paulo: Manole, 2006. p.175-218.

PANG, D.; NEMZEK, W.R.; ZOVICKIAN, J. Atlanto-occipital dislocation: part 1-normal occipital condyle-c1 interval in 89 children. Neurosurgery., v.61, p.514-521, 2007a.

PANG, D.; NEMZEK, W.R.; ZOVICKIAN, J. Atlanto-occipital dislocation-part 2: the clinical use of (occipital) condyle-c1 interval, comparison with other diagnostic methods, and the manifestation, management, and outcome of atlanto-occipital dislocation in children. Neurosurgery., v.61, p.995-1015, 2007 b. 
PRZYBYLSKI， G.J.; CLYDE， B.L.; FITZ, C.R. Craniocervical junction subarachnoid hemorrhage associated with atlanto-occipital dislocation. Spine., v.21, p.1761-1768, 1996.

RYLANDER, R.; ROBLES, J.C. Diagnosis and treatment of a chronic atlanto-occipital subluxation in a dog. J. Am. Anim. Hosp. Assoc., v.43, p.173-178, 2007.
STEFFEN, F.; FLUECKIGER, M.; MONTAVON, P.M. Traumatic atlanto-occipital luxation in a dog: associated hypoglossal nerve deficits and use of 3-dimensional computed tomography. Vet. Surg., v.32, p.411-415, 2003. 